\title{
Modeling of a lay-flat plastic hose extrusion process
}

\author{
E. Nordgård-Hansen ${ }^{1}$ R. Schlanbusch ${ }^{1}$ T. Sørensen ${ }^{2}$ \\ ${ }^{1}$ Teknova AS, Tordenskjolds gate 9, 5 etg., NO-4612 Kristiansand, Norway. E-mail: \{enh,rsc\}@teknova.no \\ ${ }^{2}$ Fenner Mandals, Nordre Banegate 26, NO-4515 Mandal, Norway. E-mail: sorensen@mandals.com
}

\begin{abstract}
Many complex processes have a low degree of automation, and oftentimes important quality information is only available hours or even days after the production is completed. This article shows how multivariate design and response surface modeling were applied to a lay-flat plastic hose extrusion process in a full-scale experiment. Clear quantitative relationships were found, which to a large degree match existing qualitative process understanding. For instance, it was quantified how adhesion improves with increased extrusion screw speed and extrusion head temperature. The results can readily be used to inform the operators in real-time of important quality parameters of the hose currently under production. The clear results also indicate that increased process automation is achievable.
\end{abstract}

Keywords: Extrusion, Response surfaces, Experimental design

\section{Introduction}

\subsection{Background}

In the past few years, Industrie 4.0 and digitalization have become buzz-words that turn up whenever industry is discussed. Many small or traditional companies with small or non-existing research departments find it hard to allocate resources to move their processes into concepts like "Cyber-physical systems" or "Digital twins". The purpose of this article is to show how statistical modeling techniques can be applied to a complex process having a low degree of automation. It is shown how useful results can be obtained even with affordable effort, and it is discussed how one might develop the method further towards a more automated process.

The process studied is a key production step for Fenner Mandals, a company located in Mandal on the south-western coast of Norway. The company was founded in 1775 as part of the 1st industrial revolution, and has survived through the 2 nd and 3rd industrial revolution due to continuous development of process technology and changes of product portfolio. Today the most important product is lay-flat hoses. This is a soft composite hose where a circular weave is covered with a rubber or thermoplastic material. Fire hoses are typical examples. The weave provides the strength to the hose and the cover material protects and makes the hose fluid proof.

\subsection{The lay-flat hose extrusion process}

Fenner Mandals is a pioneer producer of lay-flat hoses through an in-house developed trough-the-weave extrusion process. In-house produced looms produce circular weaves that are simultaneously covered at the inside and at the outside by a thermoplastic (TP) or a rubber material: Extruders melt TP or rubber and force it into an extruder head by a screw. While the head is filled with TP, a circular weave is dragged through the head at 90 angle to the screw. In-house designed tools then distribute the TP as a mm-thin layer inside, within and outside the weave. Figure 1 shows a picture from the 
extrusion process. The weave enters the extruder head from the right and leaves the extruder as a hose with added TP or rubber to the left. Fixed tool sizes limit the possibilities for extruder head automation.

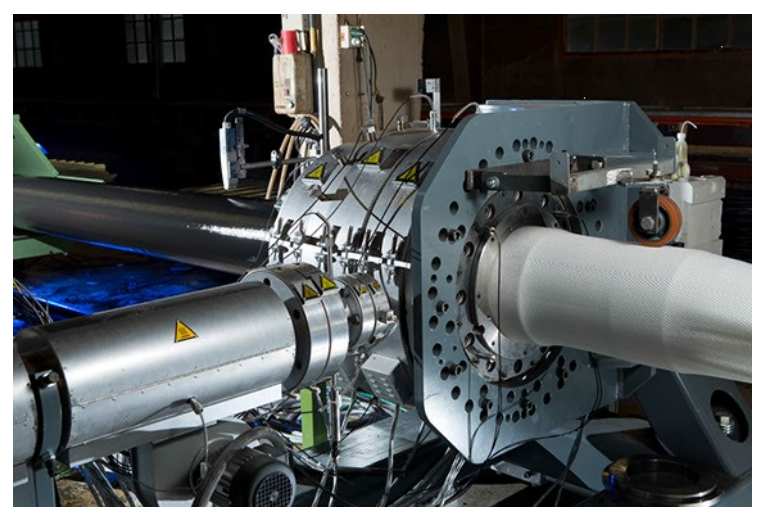

Figure 1: A picture from the extrusion process at Fenner Mandals

\subsection{Modeling the extrusion process}

A thorough, general description of the extrusion process is given by Chung (2011), and extruder modeling is also available in ANSYS (2017).

Different authors have considered various detailed aspects of extruder modeling. Abeykoon et al. (2014) show the importance of knowing the radial temperature distribution in an extruder, and they suggest a model for this. Bereaux et al. (2009) on the other hand, focus on the pressure distribution through the screw, and the resulting production rate.

Response surface modeling is frequently used for extruder processes, see for instance Lebaal et al. (2010) and Formela and Bogucki (2014). Extrusion of plastic and cereals have much in common, and response surface modeling for cereal extrusion is described by Kowalski et al. (2016). In building these surfaces, experimental design is crucial, and a discussion of practical aspects for multivariate experiments, where theory and practice do not always match, is given by Jarrett (2017).

Still, the woven filament in the hoses produced at Mandals complicate the situation, so that specific modeling of the Mandals process is required. In the current article, we first present the chosen methodology for testing and modeling, before the results are shown and discussed. We conclude with a note on further modeling work and possible applications of the models.

\section{Method}

In order to quantify process insight from existing measurements, the response surface method was chosen. An alternative was detailed physical modeling, which would be a comprehensive task for the whole process from extruder screw inlet to quality measurements, and which would also have required new measurements for verification.

\subsection{Experimental}

Input variables were chosen based on know-how of what controls the most important quality and process parameters. In cooperation with Mandals, it was decided to use a two-level factorial design with a center point and two replications, as shown in Table 1. Actual values are not given, since this is considered Fenner Mandals' proprietary information. Each input variable was varied to get the largest possible variation while keeping the process stable. The factors are listed below, and their placement in the process is sketched in Figure 2.
A Temperature set point
B Screw speed
C Pull speed

D Weave pre-drying position

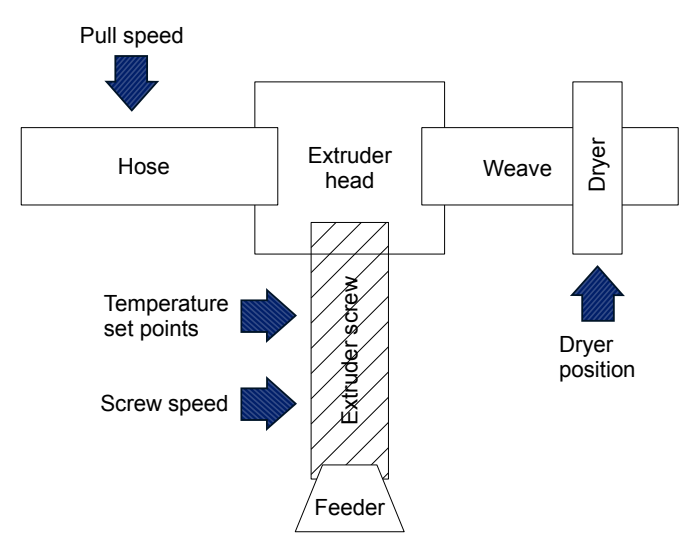

Figure 2: Sketch of input variable position

The factor A: Temperature set point is actually a group of set points. It was used to control 5 different temperatures; the extruder screw has 4 zones where the temperature set points can be set independently. In addition, there is a separate temperature set point for the extruder head. In the experiments, not all of these set point values were identical, but $A=-1$ signifies 
Table 1: Experimental design

\begin{tabular}{crrrr}
\hline Experiment no. & A & B & C & D \\
\hline 1 & -1 & -1 & -1 & -1 \\
2 & -1 & -1 & 1 & 1 \\
3 & -1 & 1 & -1 & 1 \\
4 & -1 & 1 & 1 & -1 \\
5 & 1 & -1 & -1 & 1 \\
6 & 1 & -1 & 1 & -1 \\
7 & 1 & 1 & -1 & -1 \\
8 & 1 & 1 & 1 & 1 \\
9 & 0 & 0 & 0 & 0 \\
\hline 10 & -1 & -1 & -1 & -1 \\
11 & -1 & -1 & 1 & 1 \\
12 & -1 & 1 & -1 & 1 \\
13 & -1 & 1 & 1 & -1 \\
14 & 1 & -1 & -1 & 1 \\
15 & 1 & -1 & 1 & -1 \\
16 & 1 & 1 & -1 & -1 \\
17 & 1 & 1 & 1 & 1 \\
18 & 0 & 0 & 0 & 0 \\
\hline
\end{tabular}

the lower set point value and $\mathrm{A}=1$ signifies the upper set point value for all 5 temperatures in the group.

The center points represent typical set point values, and are not in the exact center of the design. The reason for this off-center position was that typical set point values are not in the center of the relevant stable variation range for all input variables. Note that the weave pre-drying position $\mathrm{D}$ is confounded with the three-factor interaction between the other factors, ABC. This makes the design a $2^{4-1}$ two-level design with two replications and two center points. The experiments took place in the actual production line. The tests used raw material and production time that could have been spent making salable products. As a consequence, it was not possible to randomize the order of the experiments. Instead it was necessary to change the input variable that required most time to reach steady state least frequently.

The tests were conducted at Mandals' location in Mandal Friday February 10th 2017, while the laboratory results were received during the following weeks. For the values registered during the tests, the final value for each experiment was used, representing (close to) steady state.

The defined result variables are listed below:

- Mass pressure measured between the screw and extruder head. This was registered during the experiments. It is an important variable as it is a measure of process stability, and because the equipment has safety limits regarding pressure.
- Electric current to the extruder, registered during the experiments. This is an indirect measurement of the viscosity of the plastic or rubber, and also an important part of the process energy consumption.

- Total hose thickness, measured in the laboratory at four positions equally distributed around the hose circumference according to ISO standard 4671. The hose should be thick enough to cover the weave and withstand abrasive environments. On the other hand, a too thick hose adds material cost and unnecessary weight.

- Coating thickness, measured in the laboratory at four positions equally distributed around the hose circumference.

- Adhesion, measured in the laboratory at four positions equally distributed around the hose circumference according to ISO 8033. Only the average of the two positions not influenced by hose flattening was used.

- Abrasion resistande accordning to BS 6391, laboratory measurements of mass reduction per standardized abrasion cycle.

\subsection{Modeling}

All input variables were scaled, resulting in values of $-1,1$, and 0 . This means the relative effect of each variable can be compared directly, independent of the chosen measurement unit. The result variables were not scaled, since they were not compared to each other.

For each result variable, the contrasts were used to find significant input variables, including any important interactions. Since the dryer position was confounded with the three-factor interaction between the other three input variables, this position was not considered as a separate input variable, but only considered if the three-factor interaction was found to be statistically significant.

Based on these results, multiple regression was used to find response surfaces. These were then analyzed, and if not satisfactory, improvements were tried out, based on process understanding and on input data details. The center points were not used in the regression, but rather used to evaluate the assumption of plane response surfaces.

The statistical analysis presented here was performed using the python package Statsmodels Seabold and Perktold (2010). 
Table 2: Model results

\begin{tabular}{ll}
\hline Result & Depends on \\
\hline Mass pressure & Temperature set point and screw speed \\
Current & Temperature set point and screw speed \\
Average total thickness & Screw speed and pull \\
Coating thickness & Screw speed, pull and temperature set point \\
Adhesion & Screw speed, pull and extruder head inner temperature \\
Abrasion resistance & No dependency found \\
\hline
\end{tabular}

Table 3: Statistical model properties

\begin{tabular}{lcccc}
\hline & $\mathrm{R}^{2}$ & Adjusted $\mathrm{R}^{2}$ & Cross validation $\mathrm{R}^{2}$ & Prob(F-statistic) \\
\hline Mass pressure & 0.94 & 0.93 & 0.91 & $9.8 \cdot 10^{-09}$ \\
Current & 0.97 & 0.96 & 0.95 & $3.4 \cdot 10^{-10}$ \\
Average total thickness & 0.99 & 0.99 & 0.98 & $9.1 \cdot 10^{-14}$ \\
Coating thickness & 0.99 & 0.98 & 0.98 & $1.4 \cdot 10^{-10}$ \\
Adhesion & 0.93 & 0.91 & 0.85 & $5.0 \cdot 10^{-07}$ \\
Abrasion resistance & - & - & - & - \\
\hline
\end{tabular}

\section{Results}

A summary of all models is given in Table 2, while some key statistical properties are listed in Table $3 . \mathrm{R}^{2}$ is a measure of how well the model fits the data used to generate it, while Adjusted $\mathrm{R}^{2}$ is a value for $\mathrm{R}^{2}$ which is decreased when a large number of input variables is used. Cross validation $\mathrm{R}^{2}$ resulted from leave-one-out cross validation, and is thus a measure of how good the model is to predict samples that were not used to generate the model. Prob(F-statistic) is the probability that the current model is the result of purely random variation in the data. A more detailed explanation and description of the results for each result variable is given in the following.

\subsection{Mass pressure}

As seen in the normal plot in Figure 3, two input variables influence the mass pressure, A: Temperature set point and B: Screw speed. The red line represents the relation one would expect if the contrasts were normally distributed. The resulting linear response surface is shown in Figure 4. The blue dots represent the data in the training set, while the red dots, partly hidden beneath the surface, represent the center points.

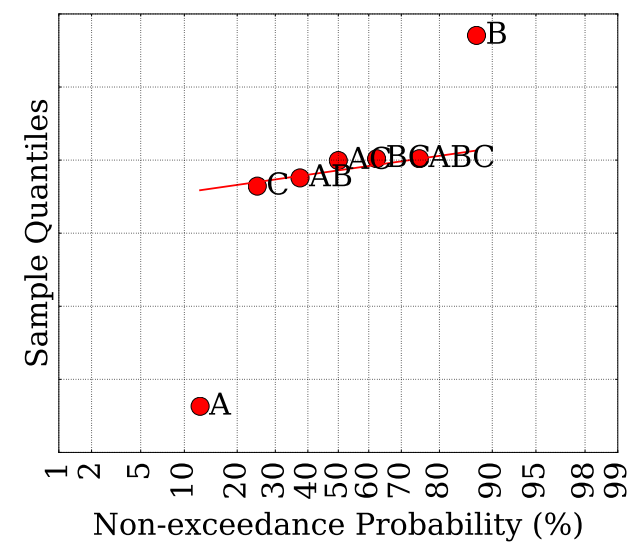

Figure 3: Mass pressure contrasts

\subsection{Electric current}

As seen in the normal plot in Figure 5, the current to the extruder screw is mainly influenced by A: Temperature set point, where higher temperature gives lower current. In addition, the interaction between 


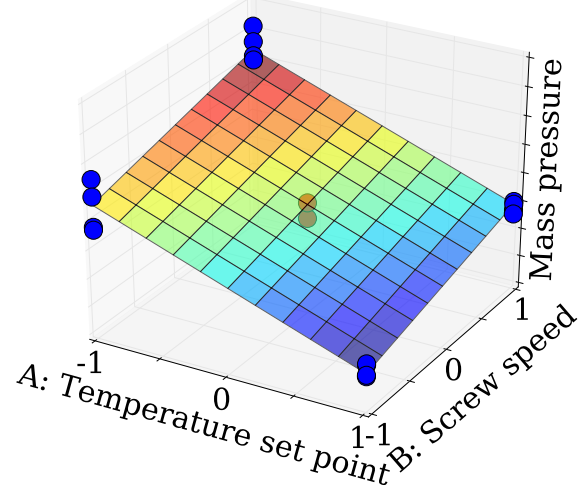

Figure 4: Mass pressure response surface

B: Screw speed and A: Temperature set point is important, where increased screw speed will reduce the negative temperature effect. The resulting response surface is shown in Figure 6.

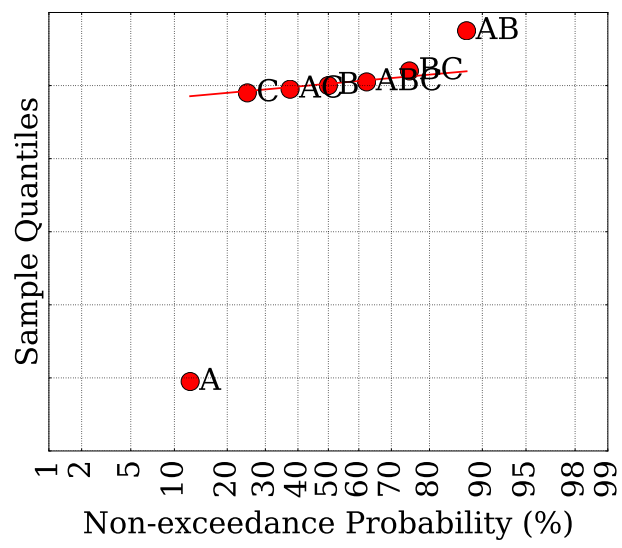

Figure 5: Electric current contrasts

\subsection{Average total thickness}

The main input variables influencing the average total thickness is B: Screw speed and C: Pull, as seen from Figure 7. Simple physical modeling indicates that the average total thickness should depend on the screw speed relative to the pull speed. When making the response surface model, it was found that a slightly better model fit was obtained by taking advantage of this fact. The resulting response surface is shown in Figure 8.

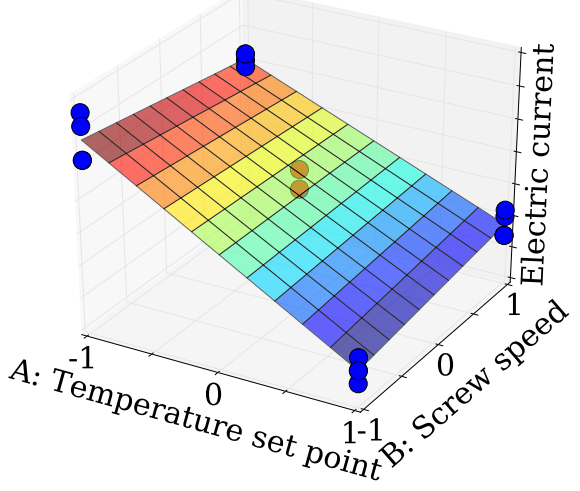

Figure 6: Electric current response surface

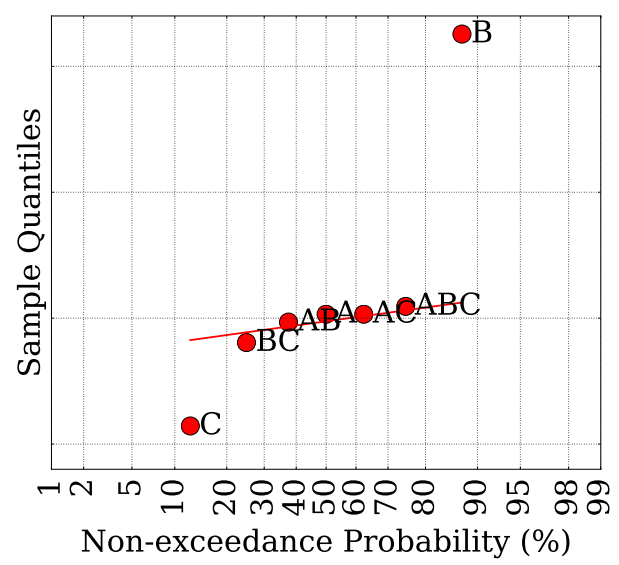

Figure 7: Average total thickness contrasts



Figure 8: Average total thickness response surface 


\subsection{Coating thickness}

This property is the hose thickness on the exterior of the woven filament. A comparison of this thickness and the total thickness thus indicates how the filament is positioned within the hose. As seen from Figure 9, it depends on B: Screw speed, and possibly on C: Pull. Similar to the total thickness, simple physical modeling indicates that the coating thickness should depend on the screw speed relative to the pull speed. The resulting response surface is shown in Figure 10.

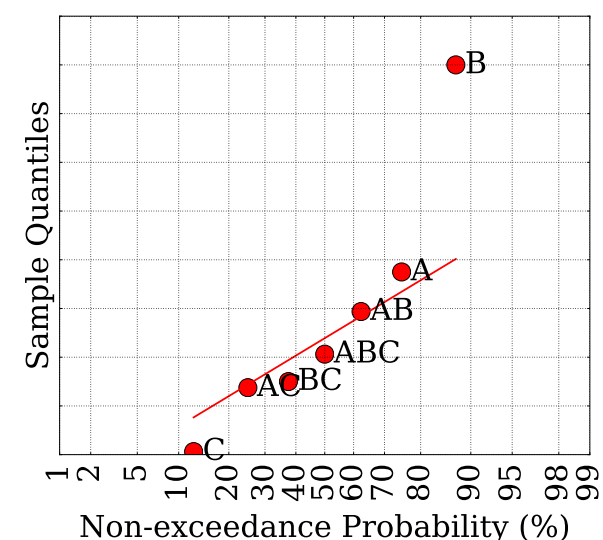

Figure 9: Coating thickness contrasts

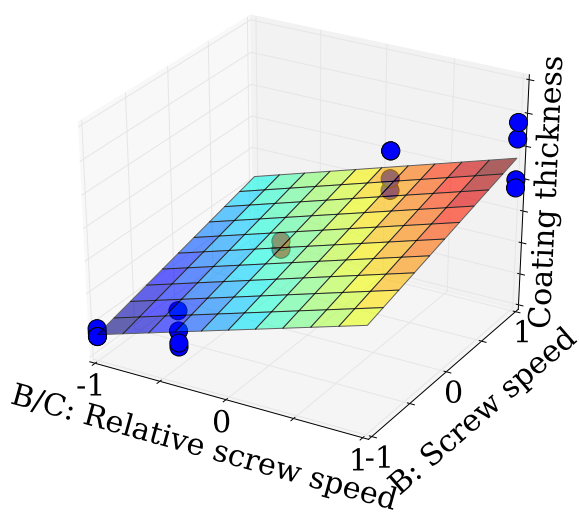

Figure 10: Coating thickness first response surface

The resulting fit is not as good as for the total thickness. Including A: Temperature set point and the interaction between this set point and the relative screw speed in the model resulted in statistically significant regression coefficients, and gave an improved $\mathrm{R}^{2}$ of 0.99 .

Coating thickness was now found to
- Increase with increasing screw speed

- Decrease with increasing pull speed

- Increase with increasing temperature set point

In Figure 11, a comparison of measured and estimated values is shown. Similar to for the 3D plots, the red dots represent the center points. The dashed diagonal line represents estimated data being identical to the measured data.

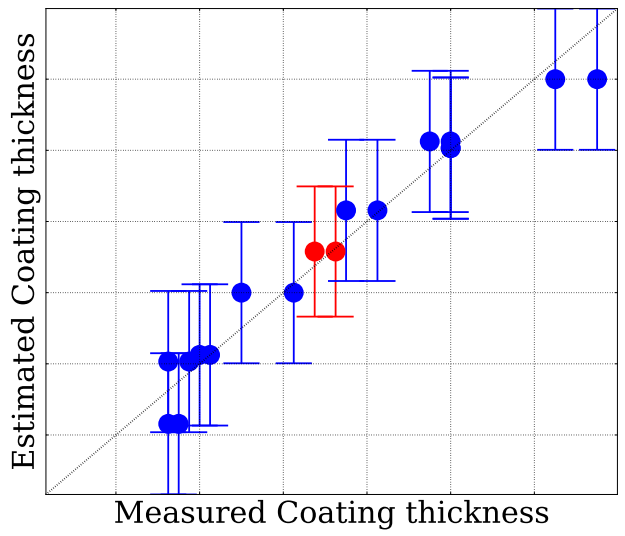

Figure 11: Measured and estimated coating thickness

\subsection{Adhesion}

As seen in Figure 12, the main input variables influencing the adhesion, are B: Screw speed and C: Pull. However, as seen from Figure 13, the corresponding response surface does not fit the measurements very well. This is also illustrated by the low value for $\mathrm{R}^{2}$ of 0.75 .

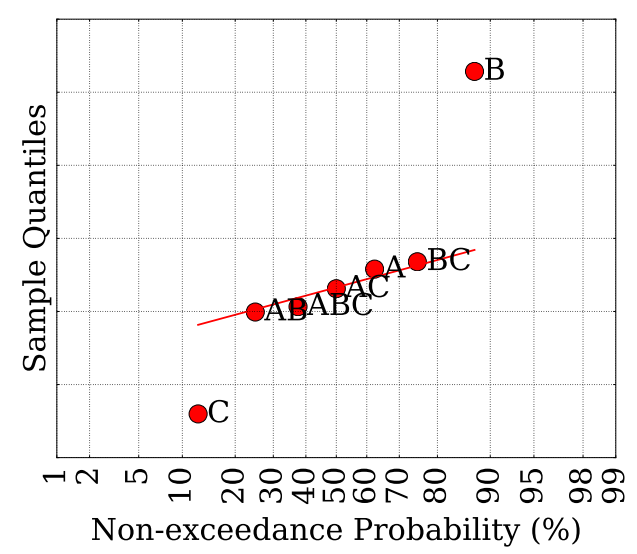

Figure 12: Adhesion contrasts 


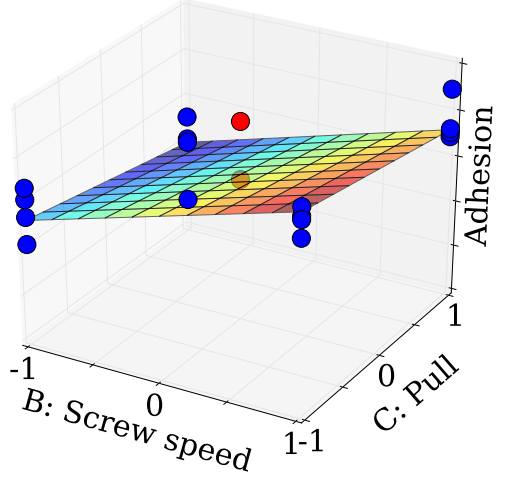

Figure 13: Adhesion first response surface

Looking deeper into the data from the experiments, it was seen that three of the internal temperatures varied strongly between the two replications. These three temperatures were then taken into the regression model in addition to screw speed and pull. One of these, an extruder head inner temperature, resulted in a statistically significant regression coefficient, and the corresponding $\mathrm{R}^{2}$ increased to 0.93 .

Adhesion was now found to

- Incease with increasing screw speed

- Decrease with increasing pull

- Increase with increasing extruder head inner temperature

In Figure 14, a comparison of measured and estimated values is shown.

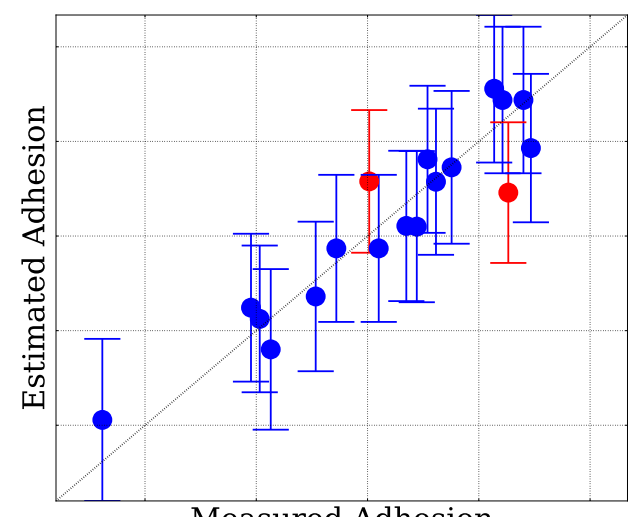

Figure 14: Measured and estimated adhesion

\subsection{Abrasion resistance}

No relation was found between the input variables and the measured abrasion resistance, as indicated in Figure 15. The number of abrasion cycles required to expose the weave was found to increase consistently with increasing coating thickness. This fact also indicates that the abrasion resistance qualities of the plastic material were not influenced by the changes made to the input variables.

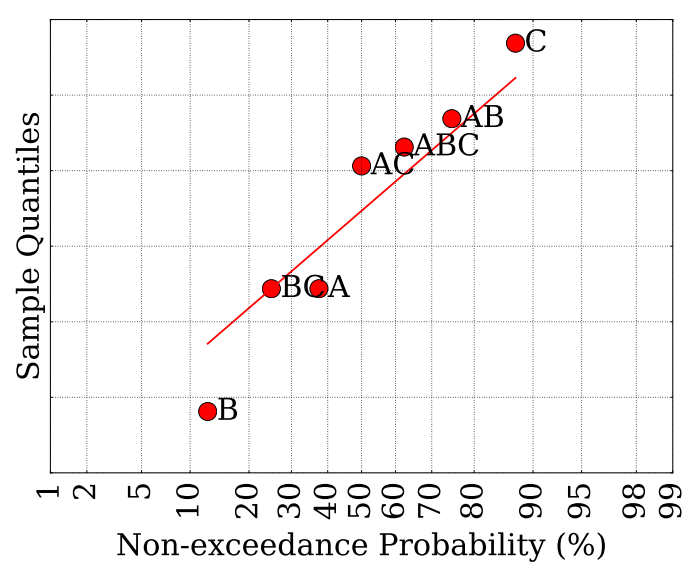

Figure 15: Abrasion resistance contrasts

\section{Discussion}

\subsection{Model validation}

\subsubsection{Normality}

In the statistical evaluation of the regression model it is assumed that the residuals after the modeling are normally distributed. This is most often the case for the models described here. An example is shown in Figure 16, where each blue dot represents a measurement in the training set, and the red line represents the relationship expected if the residuals followed a normal distribution. In Figure 17, the residuals are plotted against the predicted values. Again, no clear pattern is observed.

Initially, the average total thickness was modeled with a linear dependency on both screw speed and pull speed, without considering the relative speed. The residuals then showed a clear deviation from a normal distribution, which resulted in a more careful model formulation.

Since the sign of the effects depends on how the two levels are coded, it is common practice to use half normal plots of the effects' absolute values to determine 


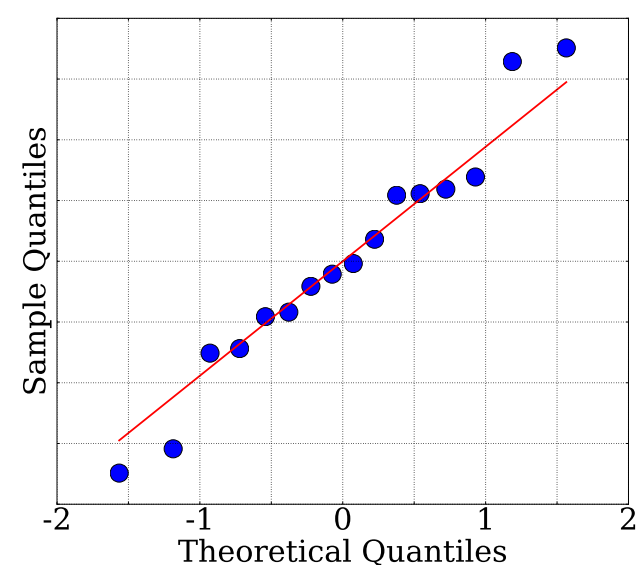

Figure 16: Distribution of residuals after modeling for mass pressure

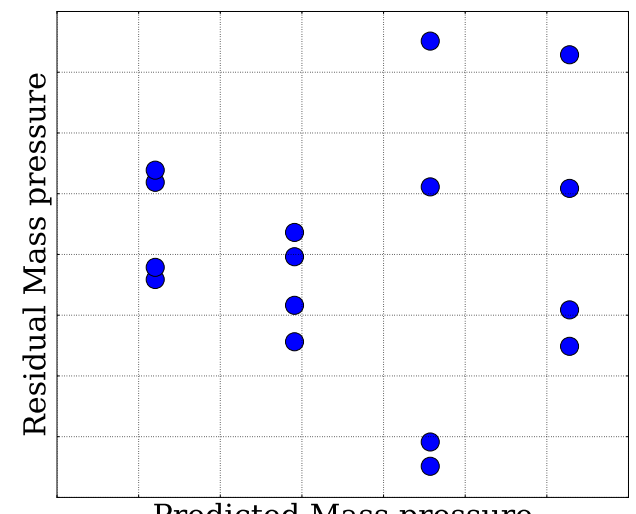

Predicted Mass pressure

Figure 17: Residuals as a function of predicted values after modeling for mass pressure which effects are significant. In the current study, the full normal plot was used, since the effects are coded so that the sign actually contains information.

\subsubsection{Predictive strength}

The adjusted $\mathrm{R}^{2}$ is a measure of $\mathrm{R}^{2}$ adjusted for the number of variables chosen. Using a large number of variables to explain a small number of tests would result in a large distance from the model's $\mathrm{R}^{2}$. The numbers in Table 3 do not indicate any such over-fitting.

$\mathrm{R}^{2}$ shows how good the model describes the data used to make the model. With a total of 18 experiments, of which 16 were used directly in the model building and 2 were used for evaluation of curvature, there is no room for dividing the results in a training set and an evaluation set. Instead, leave-one-out cross validation was used, resulting in the values for $\mathrm{R}^{2}$ from cross validation given in Table 3. The fact that these values are not far lower than the $\mathrm{R}^{2}$ from the modeling indicates that no single test strongly influenced the model.

As far as it is possible to conclude from this low number of tests, the models can be expected to predict the quality parameters well for input variables within the range used in the experiments.

\subsubsection{Plane response surfaces}

The models were made from the corner points, and the center points were used to evaluate if the response surfaces should actually be curved. As seen from the response surface plots and from Table 4, there is no reason to abandon the assumption of plane surfaces. All numbers in the table are given relative to the corresponding difference between the center points. For further modeling of mass pressure and thickness, one should still keep in mind that a curved surface may be required if the region of variation is increased. The two center points alone do not give enough information to robustly determine any second degree regression coefficients.

\subsection{Process dynamics}

The basic idea of the current study was to consider the effect of the systematically varied input variables by waiting until stationary state was reached for each set point combination. It was observed that some measurements either took very long time to reach stationary state, or did not depend directly on the set points. One example is the extruder head inner temperature, which is part of the adhesion model. If the models developed here are later used for process control, process dynamics must also be considered. 
Table 4: Surface curvature

\begin{tabular}{cccc}
\hline Model & Distance from surface & $\begin{array}{c}\text { Mean standard deviations } \\
\text { between replications }\end{array}$ & $\begin{array}{c}\text { Distance between } \\
\text { center points }\end{array}$ \\
\hline Mass pressure & 1.5 & 0.7 & 1.0 \\
Current & 0.3 & 0.8 & 1.0 \\
Average total thickness & 1.0 & 0.6 & 1.0 \\
Coating thickness & 1.0 & 0.7 & 1.0 \\
Adhesion & 0.2 & 0.4 & 1.0 \\
Abrasion resistance & - & - & - \\
\hline
\end{tabular}

\subsection{More than one measurement of each response}

Some of the response variables, e.g. total thickness, is the result of two or four measurements of each quantity, i.e. two or four positions along the circumference on the hose. To take this into the model, each point may be weighted by the inverse standard deviation at each point. Points with large differences between the measurements are thus given less weight in the regression analysis.

For total and coating thickness this procedure has only minor influence on the resulting model. On the other hand, weighted modeling gives residuals that are closer to normally distributed, which indicates that the deviations from normality was related to measurements with a large uncertainty. For adhesion, weighting by the inverse standard deviation strongly reduced the model's predictive strength, while several tests for normality failed. Looking into the data, it was seen that tests 4,5 , and 8 exhibited large standard deviations in both replications, thus removing important information from the model when these samples were down-weighted.

In general it is recommended to use the inverse standard deviation weighting with caution, especially when only a few tests are performed.

\subsection{Physical interpretation}

While data-based modeling can give new insight into the physical processes, it is equally important that the resulting models can be explained physically.

All relationships shown in Table 2 fit well with an intuitive process understanding.

The power requirement to the heaters along the extruder screw were not registered during the experiments. Thus it is not known if the decrease in current to the extruder screw obtained by increased temperature set points is balanced by increased power requirement to the heaters.

Even though the temperature set point did not ex- hibit significant contrast on the coating thickness in Figure 9, it did exhibit significant regression coefficients, where increased temperature led to increased coating thickness. This is contrary to what was expected, since high temperature plastic has lower viscosity and thus moves more easily from the weave exterior to the interior. As discussed for adhesion, the temperatures measured inside the extruder head did not all vary strongly with temperature set point. Thus, the viscosity of the plastic inside the extruder head may not vary strongly either. On the other hand, the pressure measured at the end of the extruder screw did increase strongly with decreased temperature set point, which may aid in pushing the molten plastic through the weave. Clearly, further work is required to obtain a detailed understanding of how plastic penetrates the weave within the extruder head.

It may seem surprising that the adhesion depends on a measured temperature, and not on the temperature set point. The explanation is that the extruder head inner temperature describes a heating during the day to a near constant temperature, rather than a response to a change in the temperature set point. This temperature is measured close to where the molten plastic penetrates the woven filament. The inclusion of this variable thus gives intuitive physical sense as well. It is interesting to note that high adhesion is measured at conditions that also give high hose thickness. This fits well with the notion that measured adhesion as a result mainly of binding between the plastic and the woven filament, but also of coating thickness.

The lack of modeling results for abrasion resistance can be explained by this property depending on the chemical properties of the hose, such as plastic type, additives, etc. For the extrusion process to change this, the temperatures and other process variables would have to vary far beyond the normal operational window.

For the specific hose and variables tested it can be concluded that

- Increased process temperatures result in 
- Reduced mass pressure due to reduced viscosity

- Reduced current requirement to the extruder screw, also due to reduced viscosity

- No change of total hose thickness, which was unexpected and contrary to current knowhow

- Increased inner extruder heat temperature results in improved adhesion

- Increased screw speed results in

- Increased mass pressure

- Reduced temperature influence on the current requirement

- Increased hose thickness, total as well as coating thickness

- Better adhesion

- Increased pull speed results in

- Reduced thickness, total as well as coating thickness

- Reduced adhesion

- Dryer position is of negligible importance

\subsection{Further work}

A few results were unexpected, such as the coating thickness increasing with increased extruder temperature and the temperature set points not having any strong influence on the total hose thickness. Further investigations are required to verify and understand these findings.

This study was conducted using one specific kind of hose, i.e. one extruder, one woven filament, and one kind of plastic. To expand to other hose types, similar experiments must be performed for these, including varying the filament properties. Some models may be used for several different kinds of hoses, e.g. by scaling with the hose diameter, but such assumptions must be thoroughly verified before they are used. Preliminary tests done on a similar hose with a different diameter but from the same kind of plastic do support this kind of scaling.

The relative gain array (RGA) as described by Skogestad and Postlethwaite (2005) is an interaction measure between input-output pairs. RGA analysis may thus be used as a tool for further work.

\subsection{Applications}

Since clear relationships are found between input variables and quality parameters, a simple system may be implemented for the operator to see real-time expected values of some critical properties, based on the current process values. This kind of system may be introduced for one type of hose at the time, meaning that the system can be useful even before all kinds of combinations are modeled.

Dynamic versions of the models presented here may be used for automatic process control.

\section{Conclusion}

Clear quantitative relationships were found between important quality parameters and the three input variables extrusion screw speed, extruder temperature and extruded hose pull speed. To a large degree, the relationships match existing qualitative process understanding. For instance, it was quantified how adhesion improves with increased extrusion screw speed and extrusion head temperature. On the other hand, it was found that total hose thickness was governed by extruder screw speed and extruded hose pull speed, while it was not significantly influenced by the extruder temperatures.

The results can readily be used to inform the operators real-time of important quality parameters of the hose they are currently producing. The clear results also indicate that increased process automation is achievable.

\section{Acknowledgments}

The project was partly funded by the Regional Research Funds of Agder, Norway, project 268873.

The authors would like to thank Fenner Mandals for providing raw materials, production time and operators for a seven-hundred-meter long hose, and also for contributing with know-how regarding lay-flat hose extrusion, and performing the laboratory measurements.

\section{References}

Abeykoon, C., Martin, P., Li, K., and Kelly, A. Dynamic modelling of die melt temperature profile in polymer extrusion: Effects of process settings, screw geometry and material. Applied Mathematical Modelling, 2014. 38(4):1224-1236. doi:10.1016/j.apm.2013.08.004.

ANSYS. ANSYS. 2017. URL www.ansys.com/ products/fluids/ansys-polyflow. 
Bereaux, Y., Charmeau, J.-Y., and Moguedet, M. A simple model of throughput and pressure development for single screw. Journal of Materials Processing Technology, 2009. 209(1):611-618. doi:10.1016/j.jmatprotec.2008.02.070.

Chung, C. Extrusion of Polymers. Theroy and Practice. Hanser, 2011.

Formela, K. and Bogucki, M. Use of response surface methodology in characterization of properties of recycled high density polyethylene/ground tire rubber compositions. Polimery/Polymers, 2014. 59(6):488494. doi:10.14314/polimery.2014.488.

Jarrett, R. Does theory work in practice? two case studies. Quality Engineering, 2017. 29(1):141-159. doi:10.1080/08982112.2016.1211894.

Kowalski, R., Medina-Meza, I., Thapa, B., Murphy, K., and Ganjyal, G. Extrusion processing characteristics of quinoa (chenopodium quinoa willd.) var. cherry vanilla. Journal of Cereal Science, 2016. 70:91-98. doi:10.1016/j.jcs.2016.05.024.

Lebaal, N., Puissant, S., and Schmidt, F. Application of a response surface method to the optimal design of the wall temperature profiles in extrusion die. International Journal of Material Forming, 2010. 3(1):4758. doi:10.1007/s12289-009-0416-x.

Seabold, S. and Perktold, J. Statsmodels: Econometric and statistical modeling with python. In 9th Python in Science Conference. 2010.

Skogestad, S. and Postlethwaite, I. Multivariable feedback control : analysis and design. Wiley, 2 edition, 2005. 